\section{Formación en Neuropsiquiatría: ¿Una necesidad de país?}

\section{Training in Neuropsychiatry: A National need?}

Sr. Editor: No existe en la actualidad una definición consensual de Neuropsiquiatría. No basta plantearla como "una disciplina puente entre Neurología y Psiquiatría", en tanto los dominios programáticos de ambas, especialmente de la Psiquiatría, no están claramente establecidos. Como plantean Berríos y Marková ${ }^{1}$ una definición de Neuropsiquiatría dependería del punto de vista adoptado, de los problemas analizados, del método de investigación empleado, del tipo de pacientes en cuestión o de una combinación de los anteriores. Una primera aproximación es la de Mitchell ${ }^{2}$, quien la define como "una disciplina que trata los aspectos psiquiátricos de las enfermedades neurológicas y los aspectos neurológicos de las enfermedades psiquiátricas", o la de Miyoshi y colaboradores ${ }^{3}$, que postulan que es "una neurociencia integrada de la Neurología y la Psiquiatría que tendría por objetivo investigar los síntomas psiquiátricos de los desórdenes neurológicos y las bases biológicas de los desórdenes psiquiátricos". Otros, como Yudofsky y Hales ${ }^{4}$, proponen definiciones más ambiciosas: "muchas condiciones humanas que actualmente no se consideran parte de la medicina, llegarán a constituir-gracias a los avances en neurociencias- trastornos neuropsiquiátricos". En términos globales, la Neuropsiquiatría analizaría la relación mente/cuerpo dentro del marco de las neurociencias. Cabe enfatizar que una definición de Neuropsiquiatría será hoy necesariamente provisoria.

Siguiendo a Yudofsky y Hales ${ }^{4}$, la Neuropsiquiatría es una disciplina abocada al estudio de la estricta relación entre el cerebro y las funciones cognitivas, la mente y el cuerpo, lo mental y lo físico. Su campo clínico consistiría en la evaluación y tratamiento de pacientes con enfermedades psiquiátricas o síntomas asociados a lesiones o disfunción cerebral. Se ocuparía también de los síndromes fronterizos, como los trastornos atencionales, de percepción y memoria. De este modo, proponemos definir Neuropsiquiatría como un sistema de conocimientos destinado a explicar: i) fenómenos psiquiátricos desde una óptica neurológica; ii) fenómenos neurológicos a partir de una visión psiquiátrica y/o iii) fenómenos conductuales, cognitivos y emocionales, integrando tanto las posturas teóricas psiquiátricas como las neurológicas. De esta manera, se configura como una ciencia multidisciplinaria relativa a las neurociencias. En la praxis, la Neuropsiquiatría ha permitido ligar las actividades de la Psiquiatría y Neurología, introduciendo nuevos modelos de comprensión de las enfermedades neurológicas y psiquiátricas, aspirando con ello a refinar el tratamiento de estos cuadros. Un ejemplo de lo anterior es el caso de la psicopatía, cuyo estudio desde una perspectiva neuropsiquiátrica ha contribuido sin duda a dilucidar sus bases cerebrales y postular una comprensión de los circuitos neuronales disfuncionales de otros síndromes psiquiátricos.

Los antecedentes históricos de la Neuropsiquiatría se remontan a mediados del siglo XIX, cayendo en el descrédito a comienzos del siglo $\mathrm{XX}$, probablemente en relación al surgimiento del Psicoanálisis y a la "psicologización" de la Psiquiatría ${ }^{1}$. Desde la década de 1980, ha experimentado un nuevo impulso evidenciado en la creación en 1989 del Journal of Neuropsychiatry and Clinical Neurosciences, la creación de la subespecialidad de Neuropsiquiatría en Estados Unidos, el surgimiento de la International Organization of Neuropsychiatry (ION) en 1986 y la re-organización, en 1998, de esta entidad precursora como la International Neuropsychiatric Association (INA) ${ }^{3,4}$. Dicho resurgimiento puede explicarse por tres elementos fundamentales: 1) La elevada prevalencia de las enfermedades neuropsiquiátricas y el monto de discapacidad asociada a éstas. De acuerdo a la OMS, los trastornos neuropsiquiátricos son la causa más importante de discapacidad a nivel mundial y constituyen en nuestro país la primera causa de discapacidad en ambos sexos; 2) Un paradigma neuropsiquiátrico podría disminuir el fuerte estigma asociado a las enfermedades psiquiátricas, al poner el foco en las lesiones cerebrales y procesos fisiopatológicos subyacentes ${ }^{5}$ y 3 ) Los avances en neurociencias, al permitir progresos en la comprensión de los circuitos cerebrales disfuncionales en diferentes enfermedades psiquiátricas (esquizofrenia, depresión y otras), han promovido que los conceptos neuropsiquiátricos se apliquen con mayor frecuencia en la investigación y práctica clínica psiquiátrica/neurológica.

Desde la neuropsiquiatría, surgen nuevos modelos comprensivos de los trastornos neurológicos y psiquiátricos, basados principalmente en un 
método comparativo de perfiles clínico-neuropsicológicos de pacientes con trastornos psiquiátricos y de pacientes con lesiones cerebrales, integrando los hallazgos en neuroimágenes. La Neuropsiquiatría, al integrar los avances en neurociencias así como las recientes perspectivas psiquiátricas y de la Neurología Cognitiva, ha permitido un avance trascendental en la comprensión de las enfermedades psiquiátricas, plasmado en el desarrollo de nuevo modelos fisiopatológicos.

La evaluación neuropsiquiátrica incorpora al diagnóstico psiquiátrico tradicional, herramientas complementarias como la evaluación neuropsicológica, el uso de escalas para cuantificar los trastornos del comportamiento y las neuroimágenes. Estas técnicas proporcionan importante información sobre los déficits mentales que dan forma a la vida de nuestros pacientes, documentando entonces -de manera sistemática-indicadores de diferente índole atingentes para la comprensión de cada caso y el tratamiento de diversas enfermedades.

Aunque en nuestro país no existe la formación integrada en Neurología y Psiquiatría, la Psiquiatría Biológica ha permitido introducir en la formación psiquiátrica conceptos básicos acerca de los fundamentos neurobiológicos de las enfermedades psiquiátricas. En Neurología, la formación está más centrada en el estudio de pacientes hospitalizados, lo que se traduce en una formación relativamente escasa en los aspectos cognitivos, conductuales y/o emocionales de las principales enfermedades neurológicas como las demencias, la enfermedad de Parkinson o los pacientes con secuelas de traumatismos encefalocraneanos. La carencia de una perspectiva multidisciplinaria puede afectar la calidad del diagnóstico y el tratamiento en tales trastornos, al no considerar suficientemente las facetas involucradas ni la complejidad del problema.

Una comprensión cabal de los trastornos clínicos de ambas disciplinas requeriría un diálogo permanente entre ambas y recoger los aportes de disciplinas adyacentes, como la Psicología, Filosofía, Lingüística etc. La tarea no es sencilla, pues se intenta articular disciplinas que trabajan en diferentes niveles de abstracción y generación de hipótesis. Aunque ya son perceptibles los avances en rehabilitación neurocognitiva, desde los programas informatizados a distancia hasta el tratamiento individual y grupal e, incluso, psicoterapias diseñadas para el paciente con secuelas, es indudable que la mayor parte del trabajo está por hacerse y difundirse en la comunidad de pacientes y en quienes trabajamos en el área.

$$
\begin{array}{r}
\text { Luis Alvaradol,2,3, Carlos Muñoz-Neira }{ }^{2,4, a}, \\
\text { Gricel Orellana', Andrea Slachevsky, }{ }^{2,5,6,7} \\
{ }^{1} \text { Departamento de Psiquiatría Oriente. } \\
\text { Facultad de Medicina Universidad de Chile } \\
{ }^{2} \text { Departamento de Psicología. Facultad de } \\
\text { Ciencias Sociales. Universidad de Chile. } \\
{ }^{3} \text { Servicio de Psiquiatría, Hospital del Salvador. } \\
{ }^{4} \text { Unidad de Neurología Cognitiva y } \\
\text { Demencias. Servicio de Neurología. } \\
\text { Hospital del Salvador. } \\
{ }^{5} \text { Unidad de Neurología. } \\
\text { Clínica Alemana de Santiago . } \\
\text { Educación. Universidad de Chile. } \\
{ }^{6} \text { Centro de Investigación Avanzada en } \\
\text { Edínica (Instituto de Ciencias Biomédicas) y } \\
\text { Departamento de Ciencias Neurológicas } \\
\text { Oriente, Facultad de Medicina, } \\
\text { Universidad de Chile. Santiago de Chile. } \\
{ }^{7} \text { Psicólogo. }
\end{array}
$$

Los autores declaran no tener conflictos de intereses en este documento, que fue financiado por Proyecto PIA CONICYT, CIE-05.

\section{Referencias}

1. Berrios GE, Marková IS. The concept of neuropsychiatry: a historical overview. Journal of psychosomatic research 2002; 53: 629-38.

2. Mitchell AJ. Training in neuropsychiatry: is it time to reintegrate into mainstream psychiatry? Psychiatric Bulletin 2005; 29: 361-4.

3. Miyoshi K, Morimura Y, Maeda K. Neuropsychiatric Disorders. Kyoto: Springer 2010: v-vi.

4. Yudofsky SC, Hales RE. What's new in neuropsychiatry. J Neuropsychiat Clin Neurosci 1999; 11: 1-4.

5. Jacoby A, Snape D, Baker GA. Epilepsy and social identity: the stigma of a chronic neurological disorder. Lancet Neurology 2005; 4: 171-8.

E-mail: aslachevsky@me.com 